$\mathrm{Na}$ fell in 34/60 patients (57\%) and was less likely if baseline hyponatraemia existed $(38 \%$ VS $74 \% \mathrm{p}=0.004)$. A fall of $\geq 5 \mathrm{mmol} / \mathrm{l}$ occurred in $23 \%$.

Median time to nadir $\mathrm{Na}$ was 3 days and time to recovery to pre-treatment $\mathrm{Na}$ was 6.5 days. No complications of hyponatremia were observed.

Patients with VB were more likely (vs HRS patients) to have any fall in $\mathrm{Na}$ or $\mathrm{a} \geq 5 \mathrm{mmol} / \mathrm{l}$ reduction (68\% vs $47 \% \mathrm{p}=0.1$ and $32 \%$ vs $16 \% \mathrm{p}=0.12$ respectively) but failed to reach significance.

Mortality was $22 \%$ overall and a fall in $\mathrm{Na}$ was actually associated with reduced mortality $-9 \%$ vs $34 \%(\mathrm{p}=0.01)$.

Conclusion Serum $\mathrm{Na}$ falls in $>50 \%$ receiving terlipressin and a fall $\geq 5 \mathrm{mmol} / 1$ noted in $23 \%$.

However, no significant complications occurred and a fall in serum $\mathrm{Na}$ was actually associated with improved mortality. Patients with VB treated with terlipressin trended towards a greater likelihood of $\mathrm{Na}$ reduction versus those with HRS.

Disclosure of Interest None Declared.

\section{PTU-111 DETERMINING CEILING OF CARE IN DECOMPENSATED CIRRHOSIS - RIGHT DECISIONS, RIGHT PEOPLE, RIGHT TIME}

B Hudson*, H Morrison, FH Gordon, CA McCune, PL Collins, AJ Portal. Department of Hepatology, University Hospitals Bristol NHS Trust, Bristol, UK

\subsection{6/gutjnl-2014-307263.185}

Introduction Decisions to initiate intensive care measures in patients with decompensated liver cirrhosis are often controversial, with mortality approaching $90 \%$ in cirrhotics with 3 organ failure. The 2013 NCEPOD report 'Measuring the Units', which examined alcoholic liver disease-related deaths, nonetheless found that $31 \%$ of those who stood to benefit from higher level care did not receive it. We studied escalation of care decisions and subsequent outcomes in cirrhotic patients with organ failure. Methods Consecutive patients with a diagnosis of cirrhosis admitted over a 90 day period in 2013 to the Bristol Royal Infirmary were studied. Severity of liver disease was assessed using ChildsPugh and UKELD. Organ failure was defined using SOFA (Sequential Organ Failure Assessment) criteria. Care escalation/ withdrawal decisions were assessed in respect to timing, seniority and expertise of decision maker. Outcome measures of ICU admission, mortality and instigation of palliative care were recorded.

Results 42 admissions for 37 patients (ages 16-79, 79\% male, $81 \%$ related to alcohol, 22\% Childs A, 54\% Childs B, 24\% Childs C) were scrutinised. $30 \%$ had suffered variceal haemorrhage on, or during, admission. Of 17 patients admitted in organ failure, ICU admission was requested on 8 occasions ( 6 by a hepatologist, 1 during out of hours admission, 1 following out of hours deterioration). Escalation plans had been discussed with ICU prior to the point of clinical deterioration in 50\%. 3 patients were accepted to ICU for mechanical ventilation, of which none survived. 1 patient was accepted in principle but improved clinically. 4 patients were declined ICU admission on grounds of poor prognosis, all of whom had alcoholic cirrhosis. Of this group all required non-invasive ventilation, with $75 \%$ surviving to discharge. Across the entire cohort $55 \%$ of hepatologist led "for full escalation if required" decisions were agreed in principle with ICU. 33\% of ICU decisions to withdraw care were discussed with the referring hepatologist. Of the 7 patients who died overall, 4 were on an end of life tool with appropriate palliative measures in place.

Conclusion The high survival rates in patients refused intensive care, and high mortality amongst mechanically ventilated patients highlight the complexities of predicting outcomes in this population. Despite this, discussions between hepatology and ICU regarding ceiling and withdrawal of care often did not occur until the point of clinical deterioration, risking delays to esclation of care or appropriate palliation. Strategies to ensure early escalation decisions involving senior hepatologists and intensivists should be developed to ensure appropriate care is afforded to all cirrhotic patients in a timely fashion.

Disclosure of Interest None Declared.

\section{PTU-112 ASSOCIATIONS BETWEEN HEALTHCARE RESOURCE UTILISATION AND HEALTH-RELATED QUALITY OF LIFE IN CIRRHOSIS}

1J Orr*, ${ }^{2} \mathrm{~T}$ Homer, ${ }^{2} \mathrm{~L}$ Ternent, ${ }^{2} \mathrm{~L}$ Vale, ${ }^{1} \mathrm{M}$ Hudson, ${ }^{1} \mathrm{D}$ Jones. ${ }^{1}$ Institute of Cellular Medicine, Newcastle University, Newcastle Upon Tyne, UK; ${ }^{2}$ Institute of Health and Society, Newcastle University, Newcastle Upon Tyne, UK

\subsection{6/gutjpl-2014-307263.186}

Introduction Cirrhosis is associated with impaired HealthRelated Quality of Life (HRQoL) and considerable resource use. The association between healthcare utilisation and HRQoL in cirrhosis has not been previously studied.

Methods Four HRQoL tools (SF-36v2, CLDQ, PBC-40 and PROMIS-HAQ) and a healthcare utilisation tool were completed by patients with cirrhosis. Associations between resource utilisation and HRQoL were explored; means were compared using unpaired t tests.

Results 108 patients have been recruited to the study to date with completed tools received from 73. Regular care was required by $29(40 \%)$ with $15(21 \%)$ requiring help with personal care, $12(16 \%)$ with medical care, 18 (25\%) with meal preparation and 21 (29\%) with housework. All types of care were associated with significantly impaired HRQoL across all domains of all measures tested $(\mathrm{p}<0.05)$. The total number of healthcare consultations (hospital consultations + GP consultations + nurse consultations) was also related to HRQoL with patients who had five or more consultations in a 2 month period showing significant impairment compared to patients with fewer than five consultations. Specifically there was evidence of poorer physical HRQoL with mean SF-36 Physical Component Summary (PCS): $32.5 \pm 9.0$ vs $40.6 \pm 10.6, \mathrm{p}=0.001$ and functional restriction evidenced by PROMIS-HAQ: $42.2 \pm 26.9$ vs $21.1 \pm 25.2, p=0.001$. In addition, patients with more consultations had poorer social HRQoL with SF-36 Social Functioning (SF): $31.3 \pm 12.3$ vs $40.2 \pm 12.8, \mathrm{p}=0.005$ and $\mathrm{PBC}-40$ social: $36.3 \pm 9.9$ vs $30.0 \pm 9.7 \mathrm{p}=0.01$.

Conclusion The need for any type of regular care and more frequent consultations with healthcare professionals are associated with poorer HRQoL in cirrhosis.

Disclosure of Interest None Declared.

\section{PTU-113 A REGIONAL AUDIT OF THE MANAGEMENT OF PATIENTS WITH DECOMPENSATED LIVER DISEASE}

1J Dyson*, 1,2S McPherson on behalf of North East, North Cumbria Clinical Hepatology Network. 'Liver Unit, Freeman Hospital, UK; ${ }^{2}$ Institute of Cellular Medicine, Newcastle University, Newcastle Upon Tyne, UK

\subsection{6/gutjnl-2014-307263.187}

Introduction The recent National Confidential Enquiry into Patient Outcome and Death (NCEPOD) report 'Measuring the Units' found that hospitals are missing opportunities to save the 
lives of people with alcohol-related liver disease (ARLD) by failing to provide early intervention and specialist consultant input. ${ }^{1}$ Methods We aimed to review the management of patients with decompensated liver disease in the first $24 \mathrm{~h}$ after admission to hospital. This was a region-wide audit including all Trusts in the Northern Deanery. An audit proforma was designed and data collected on consecutive admissions over a 3 month period.

Results 139 patients were included in the study; 69\% male, median age 54 years (range $26-86$ years). ARLD was the cause of liver disease in $88 \%$. The median MELD score was 19 (range 6-39) and 88\% had Child-Pugh Grade B or C disease. The commonest reasons for admission were ascites (28\%), GI bleeding (21\%), encephalopathy (19\%) and jaundice (16\%).

There was a $9 \%$ mortality rate during the admission and average length of stay was 15 days.

82 patients had clinical ascites; $62 \%$ had a diagnostic tap within $24 \mathrm{~h}$ of admission, $21 \%$ waited $>24 \mathrm{~h}$ and $17 \%$ did not have a diagnostic tap. $18 \%$ had spontaneous bacterial peritonitis (SBP).

Previous alcohol history was only documented in 43\% but current daily consumption was documented in $81 \%$. Of patients with documented current alcohol excess, 92\% received pabrinex and $94 \%$ were started on CIWA.

$99 \%$ had their renal function checked on admission. $26 \%$ had renal impairment; $28 \%$ of whom did not have all their nephrotoxins stopped. Hyponatraemia (sodium $<125 \mathrm{mmol} / \mathrm{L}$ ) was present in $9 \% ; 42 \%$ of whom did not have diuretics stopped.

27 (19\%) patients had known or suspected variceal bleeding. $19 \%$ did not receive terlipressin and 30\% did not receive vitamin K. $67 \%$ of patients had an upper GI endoscopy within $12 \mathrm{~h}$ of admission, and $78 \%$ within $24 \mathrm{~h}$.

Hepatic encephalopathy was present in $32 \%$ of patients and lactulose commenced in $98 \%$.

$17 \%$ of patients were not seen by a consultant (any speciality) within $12 \mathrm{~h}$ of admission, $7 \%$ were not seen by a gastroenterology or hepatology consultant within $72 \mathrm{~h}$ of admission and 39\% were not seen within $24 \mathrm{~h}$.

Conclusion There are clear deficiencies in the acute management of patients with decompensated liver disease across the Northern region in keeping with the findings of the NCEPOD report. The findings of this audit will be shared across the region and we are instituting a 'care bundle' to focus on the key management of these patients and guide clinicians to improve patient care. We will re-audit to assess the impact of the 'care bundle' on patient care.

\section{REFERENCE}

1 http://www.ncepod.org.uk/2013report1/downloads/Meauring\%20the\%20Units_summary\%20report.pdf

Disclosure of Interest None Declared.

\section{PTU-114 HEPATOLOGY SPECIALIST NURSE LED EXTERNAL JUGULAR VENEPUNCTURE; IS IT SAFE AND EFFECTIVE?}

LD Morris*, S Frayne, J Wadsworth, M Mansoor, Y Reddy. Department of Gastroenterology and Hepatology, East Lancashire Hospitals NHS Trust, Blackburn, UK

\subsection{6/gutjnl-2014-307263.188}

Introduction Difficult venous access is a significant problem for a proportion of patients with chronic hepatitis $\mathrm{C}$ and a history of intravenous drug use. It can impede access to treatment and cause distress for the patient. Sampling blood through external jugular venepuncture (EJV) is highly successful. This enables more patients to be assessed and treated with antiviral therapy whilst participating in clinical trials. Our aim was to assess the efficacy of an EJV service in a large district general hospital, led by a Hepatology Specialist Nurse.

Methods Data was collected prospectively, recording the number of attempts needed to successfully complete EJV. All procedures were performed by an experienced hepatology nurse. Patients who underwent EJV were invited to provide feedback on overall satisfaction.

Results Between February 2012 and October 2013, external jugular venepuncture was attempted on 130 occasions in 57 chronic hepatitis $\mathrm{C}$ patients. The mean age of the patients was 39 (range 30-61) and there were 46 males and 11 females. Genotype distribution was mostly $3 \mathrm{a}(46 \%)$ and 1a (33\%). $80 \%$ of EJV procedures $(\mathrm{n}=103)$ were performed successfully on the first attempt, rising to $92 \%$ on the second attempt. In five patients (8\%), EJV failed due to: previous neck surgery (1), vein thrombosis (3) and patient anxiety (1). No procedural complications were reported. Patient experience was $100 \%$ positive.

Conclusion External jugular venepuncture is a useful method of blood sampling from patients with chronic hepatitis $\mathrm{C}$ and difficult venous access, allowing for monitoring of their therapy. A Specialist Nurse Led EJV service is safe, effective and is well received by patients. Due to this success, a number of our patients have been enrolled in clinical trials.

Disclosure of Interest None Declared.

\section{PTU-115 IDENTIFYING NAFLD IN PATIENTS ATTENDING A LIPID CLINIC - DO NON-INVASIVE SCORING SYSTEMS FOR FIBROSIS HAVE A ROLE?}

L Wong*, M Roberts, R McCorry. Gastroenterology, University Hospital of South Manchester, Manchester, UK

\subsection{6/gutjnl-2014-307263.189}

Introduction Hyperlipidaemia is a recognised risk factor for the development of non-alcoholic fatty liver disease (NAFLD). Aspartate aminotransferase (AST):alanine aminotransferase (ALT) ratio, AST-to-Platelet Ratio Index (APRI) and Fibrosis-4 (FIB-4) scores are validated, indirect, non-invasive methods which can be employed to exclude the possibility of hepatic fibrosis in the context of NAFLD. ${ }^{1}$ The aim of this study was to apply these indices to patients attending a lipid clinic, with an elevated ALT to determine what proportion would merit further hepatology assessment.

Methods We performed retrospective analysis of patients attending a lipid clinic in a university teaching hospital from 20112013. None of the patients were under gastroenterology/hepatology follow-up. In those with elevated ALT (>30 IU/L male, >19 IU/L female ${ }^{2}$ ) we calculated the AST:ALT ratio, APRI and FIB-4 scores.

Results 130 patients were included (68 male, 62 female; mean age 54 (17-93)). Platelet data was available for 113 patients and 69 (53.0\%) had elevated ALT (52\% male). In these patients the scoring systems demonstrated an AST:ALT ratio $>0.8$ in 32 (46.4\%), APRI $>0.51$ in $20(30.0 \%)$ and FIB-4 $>1.46$ score in 14 (20.3\%). 11 (15.9\%) had high scores across all 3 indices.

Conclusion We have demonstrated that a significant proportion of patients with lipid abnormalities and raised ALT may be at risk of NAFLD with fibrosis. By using a composite of these scoring systems it may be possible to identify those who would 